\title{
Digital Assessment of Anthropometric and Kinematic Parameters for the Individualization of Direct Human-Robot Collaborations
}

\author{
Dominik BONIN ${ }^{*}$, Lukas STANKIEWICZ ${ }^{2}$, Carsten THOMAS ${ }^{3}$, \\ Jochen DEUSE ${ }^{2}$, Bernd KUHLENKÖTTER ${ }^{3}$, Sascha WISCHNIEWSKI ${ }^{1}$ \\ ${ }^{1}$ Unit Human Factors, Ergonomics, Federal Institute for Occupational Safety and Health (BAuA), \\ Dortmund, Germany; \\ ${ }^{2}$ Institute of Production Systems, TU Dortmund University, Dortmund, Germany; \\ ${ }^{3}$ Chair of Production Systems, Ruhr-Universität Bochum, Bochum, Germany
}

DOI: 10.15221/16.171 http://dx.doi.org/10.15221/16.171

\begin{abstract}
For the human-centered design of ergonomic work systems, usually population based anthropometric percentile data tables, e.g. ISO 7250 [1] are used. Due to the recent trend to complex individual and small-batch productions, there is an increasing product and process variability. Thus, the need of robots without separating devices that can work in direct interaction with humans increases (human-robot collaboration, HRC). This form of direct collaboration between humans and robots is a major challenge for a human-centered and safe workplace design. The development of sensor technology, data processing as well as the interconnectivity with collaborative robots generally enables a flexible adjustment of the robot's trajectory to the human prerequisites. Yet, to enhance the individualization of direct human-robot collaborations a more detailed knowledge of the anthropometric and kinematic profile of the employee would be beneficial. Manual anthropometric and kinematic measurements are time consuming and expensive and therefore not suitable as a standard process. To overcome this issue, the presented research project focusses on the optimization of this process by using markerless motion capturing. The processing and possible use of the captured data will be shown by the example of a use case, where the human parameters are used for a virtual simulation and optimization of a HRC-workplace. Afterwards, a self-written software tool for the Microsoft Kinect v2 sensor is presented for the digital assessment of anthropometric and kinematic human parameters. In the actual case, the anthropometric parameters are captured from a static T-Pose. For the determination of the kinematic profile the employee successively performs pre-defined max range of motion movements for each joint and degree of freedom. The motions were designed in line with the neutral-zero method [2].The data is stored in a comma separated value file. For the use with other systems it is possible to export the values with calculated offsets to the standard T-Pose. Further, preliminary results of a validation study for the digital assessment of the anthropometric parameters will be presented. The main objective of the presented work with markerless motion capturing is to enhance the digital collection of individual anthropometric and kinematic data. In addition, the possibilities and constraints for the use of these digital assessed parameters for customizable HRC-workplace designs are observed
\end{abstract}

Keywords: anthropometry, markerless motion capturing, Kinect v2, human-robot collaboration

\section{Introduction}

Due to several changes in production environments like the changing age structure and product designs with increasing product variants, more and more companies start to develop shared human-robot workplaces. One idea behind the direct human-robot-collaboration is to keep older employees at their workplace and use their knowledge, experience and sense of responsibility to ensure the quality of production processes and to stabilize them. Furthermore employees should be assisted to compensate possible age related decreasing skills like endurance, velocity, force or resistance [3]. Further, the progressive development and widespread use of digital human modeling systems (DHM-systems) facilitate the prospective humane design of new work-systems. These changes offer many possibilities, as changes in workplace design can easily be simulated with integrated ergonomic assessment modules [4]. For a common assembly workplace and a given sample population, percentile tables offer a percentage of total accommodated people which fit in a specific workplace design. In addition, modern HRC-workplaces and especially customizable robot trajectories offer the possibility to utilize a more individualized concept. Therefore it is beneficial to have some additional measures that take functional motion capacity aspects like possible range of motion (ROM) and physical constraints of the employee, as well as individual segment lengths into account. However, manual functional capacity evaluation is time consuming, needs a lot of

\footnotetext{
*bonin.dominik@baua.bund.de; +49-231-90712460; www.baua.de
} 
professional expertise and is comparatively expensive. Therefore, it is inapplicable for an efficient use, especially for small and medium sized companies. A possible remedy could be the usage of new technologies like markerless motion capturing. Previous tests with markerless motion capture technologies like the Microsoft Kinect v2 were quite promising, regarding their suitability for industrial use [e.g. 5, 6]. The main advantage over other competitive technologies is, that the technology is widely available at low prices, the setup time is comparatively low, no marker setup, additional tools or special preparation of the employee is necessary. Several scientific studies attest that the measurement accuracy is sufficient for their proposed area of application [e.g. 7, 8-10]. Nevertheless, the accuracy needs to be validated for our field of interest.

\section{Use Cases}

For further development and feasibility tests for the planning and implementation of an individually aligned HRC-assistance system, two different industrial applications were used. The individual anthropometric and kinematic parameters, including the employee's height and length of different body segments as well as the range of motion for different joints, have an impact on the ergonomic assessment of the assembly workstation. The individually adopted body postures are factored in the design of the below mentioned HRC-assistance systems.

Within the first application, a lightweight robot assists an employee during the assembly of pumps. The integrated lightweight robot will operate the screwing process of stud screws into pump houses which are positioned by the employee. During the repetitive screw process of the robot the employee is able to place the stud screws on the second side of the pump house. In the second industrial application instead of a balancing system an industrial robot assists an employee at an assembly line in the automotive industry. With the help of the robot a heavy and bulky assembly will be moved into a car body, controlled by the employee. In this case the use of the robot allows the employee to do additional assembling or checking tasks in ergonomically optimized positions.

\subsection{Use Case 1: Assisted Assembly of Pump Houses}

In the current process, the employee needs to lift the pump house into an undefined start position and places up to eight stud screws by hand. With his second hand the employee has to fix the pump house. Afterwards he guides an impact screw driver to all screws. Especially this task is physically demanding, uncomfortable and repetitive. After finishing the first side of the pump house the employee has to rotate the pump house and consistently repeats all steps. With the HRC-setup, the employee will be assisted by a lightweight robot, which executes the screw process. The setup is previously simulated with an individual DHM-system (chapter 3 ) to take into account individual parameters. In the designed HRC-optimized process the employee moves the pump house into the optimized definite work position. Afterwards the employee moves the robot, which has an automated screw driver as end effector. The employee guides the robot by hand and teaches the stud positions. Only three positions need to be teached, all the others are calculated by the controller. During the hand guided teaching process the robot is only moveable in translational level. Rotations are locked to ensure an easy teaching (figure 1 , top line). After that, the employee rotates the pump house about $90^{\circ}$ and the robot screws the studs into the pump house from the rear side. Due to this workflow, the employee is able to teach the robot in an ergonomically optimized position and to perform additional tasks at the front side of the pump house while the robot is working on the rear side (figure 1, bottom line).

\subsection{Use Case 2: Assisted Assembly in the Automotive Industry}

For the assembly line in the automotive industry a standard robot with a payload of $150 \mathrm{~kg}$ will assist the employee instead of a cost-intensive, inflexible and individual manipulator. The robot grips an assembly from an automated guided vehicle (AGV) and brings it into an ergonomically optimized presentation position. This position is previously defined by a simulation of the work system with consideration of individual anthropometric and kinematic parameters to ensure, that the assembly is held in an optimized position that doesn't force the employee into harmful body positions In addition, the employee is able to influence this position and the orientation of the assembly in order to do additional assembly tasks or quality checks. Therefore, a graphical user interface (GUI) is build up on the teach pendant of the robot controller. The movement of the robot with attached assembly can only be done in safe conditions with limited velocity and within a restricted range. These movements are controlled by a redundant safety controller. Afterwards the robot moves the assembly into the car body on a definite trajectory. This process must be initiated and controlled by the employee. 

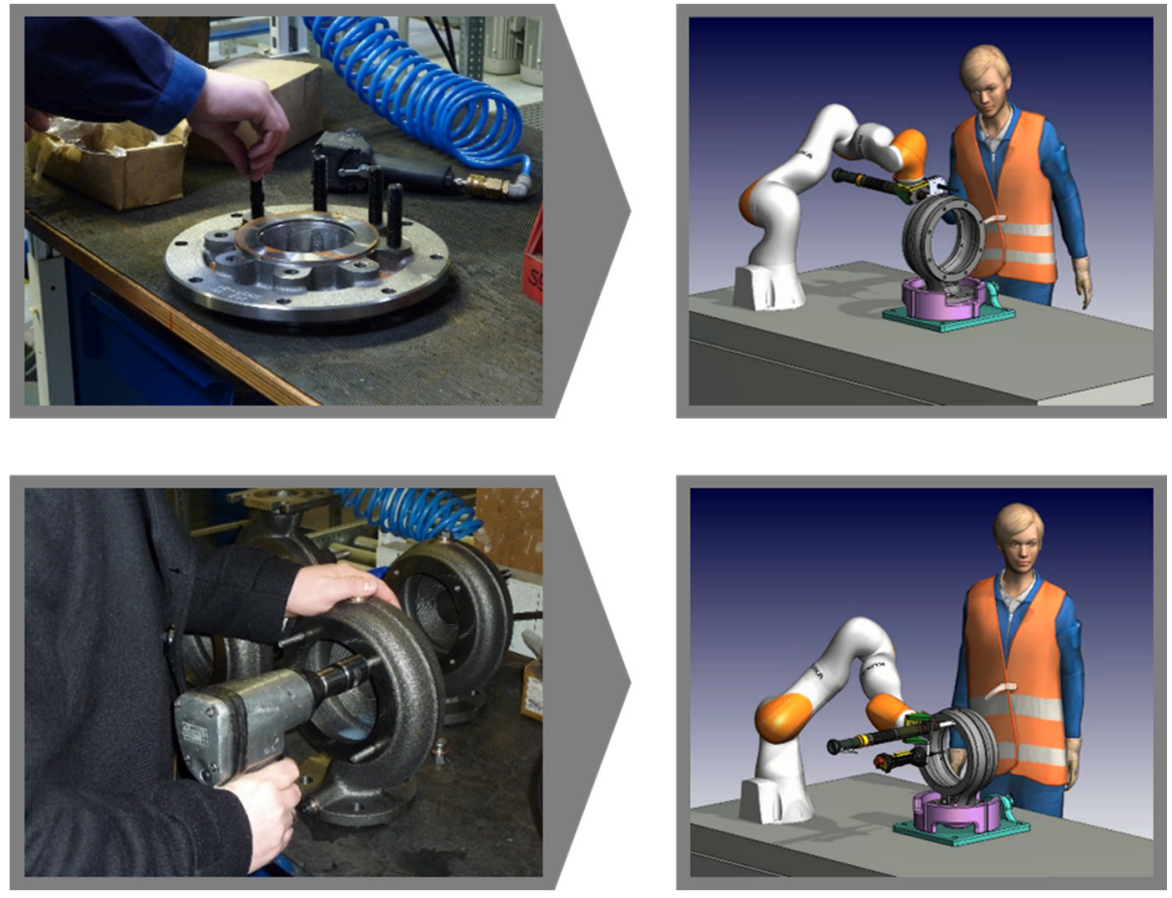

Fig. 1. Assembly of Stud Screws (left: current process, right: plant assisting process)

\section{Implementation of HRC in Industrial Applications}

The examples shown above illustrate the demand of work place adjustment for human centered work system design. The integration of robots in manual work tasks is not supposed to substitute the human workforce but to serve as an assistance system, providing support in the execution of work tasks. The overall replacement of human labor in work systems in many cases is not wanted or even feasible. In many cases robots are not able to react properly to unforeseen situations due to a restricted cognition and adaption to changing environmental influences. In that sense, the collaboration between humans and robots follows the aim to emphasize the advantages and to compensate the disadvantages of both collaborative partners [11]. HRC-systems therefore combine the flexibility of manual work systems with the strength, endurance and accuracy of robots [11]. However, the planning and design of those HRC-systems require a prospective assessment to avoid hazards and injuries of the human due to collisions with the assistance robot [12]. Defined by the product and assembly process there are tasks that include physical requirements [13] that may lead to injuries of the musculoskeletal system [14] but cannot be avoided. Robots are able to take over or assist in these tasks, like the handling of high loads or performing short-cycled work sequences. As the robot's predefined trajectories during operations in hybrid systems are hardly influenceable, a holistic simulation of the work environment including operating materials and the human factor is mandatory to ensure safety in direct HRC. Thus, the simulation needs to combine high quality forecasts of robot trajectories as well as human movements.

For this purpose an extension for the simulation software FAMOS robotic by carat robotic innovation $\mathrm{GmbH}$ was developed. FAMOS is an offline programming tool to graphically program robot's trajectories and to visualize the environment with CAD data. Specific post processors translate the designed trajectories to several supported programming languages, so that simulated trajectories can be transmitted to the robot. This ensures that the trajectories in the simulation exactly match with the real scenario trajectories. Beside the robot's motions, there has to be an exact prediction of the employee's movements in the work system in order to draw conclusions about the dynamic system behavior. For this purpose, a digital human model was integrated into FAMOS. High accuracy of the digital human model's motion data is needed to ensure that both the safety point of view in terms of injuries caused by the robot and the prevention of ergonomically questionable body postures and burdens are taken into consideration. This requires an expansion of the digital human model to an ergonomic assessment that particularly rates body postures. To ensure that process planners can use these results without an additional expert training in ergonomics, an automated assessment needs to be integrated as a plugin for the simulation tool. The ergonomic assessment used is the Rapid Entire Body Assessment (REBA), as it defines body postures by ranges of several joint angles. These can be read from the simulation by querying the angles between two body segments by determining the scalar product of the joint segment vectors. Thus, it is possible to record joint angles incessantly what allows 
an ongoing assessment of body postures during the simulation by a comparison of current joint angles with the corresponding score given by REBA. The implementation of the assessment tables by REBA for torso and legs as well as arms and hands lead to an overall score that evaluates the entire body posture (see figure 2). Thus, the process planner can detect optimization potentials during simulation runs. In addition, only little or no further information needs to be registered, allowing the user a simple and fast application. Those additional information for example may be the required physical efforts, loads or vibrations. With the possibility to adjust the human model with employee-related kinematical and anthropometrical data (see chapter 4), simulations can be run for each employee to identify individual strains by the work task. In this respect an optical motion capture system records on the one hand individual critical angles for each joint and the length of body segments on the other hand. These data are transferred to the digital human model, which thereby can be scaled and adjusted to the employee's movement restrictions. Using this method, the employees' simulation with the individualized digital human model gives very accurate movement data that can be used for precise ergonomic assessments. With the results of the ergonomic assessment, the planner is able to identify critical work tasks to determine the degree of process automation for the assistance robot and to design an individually adjusted work system.

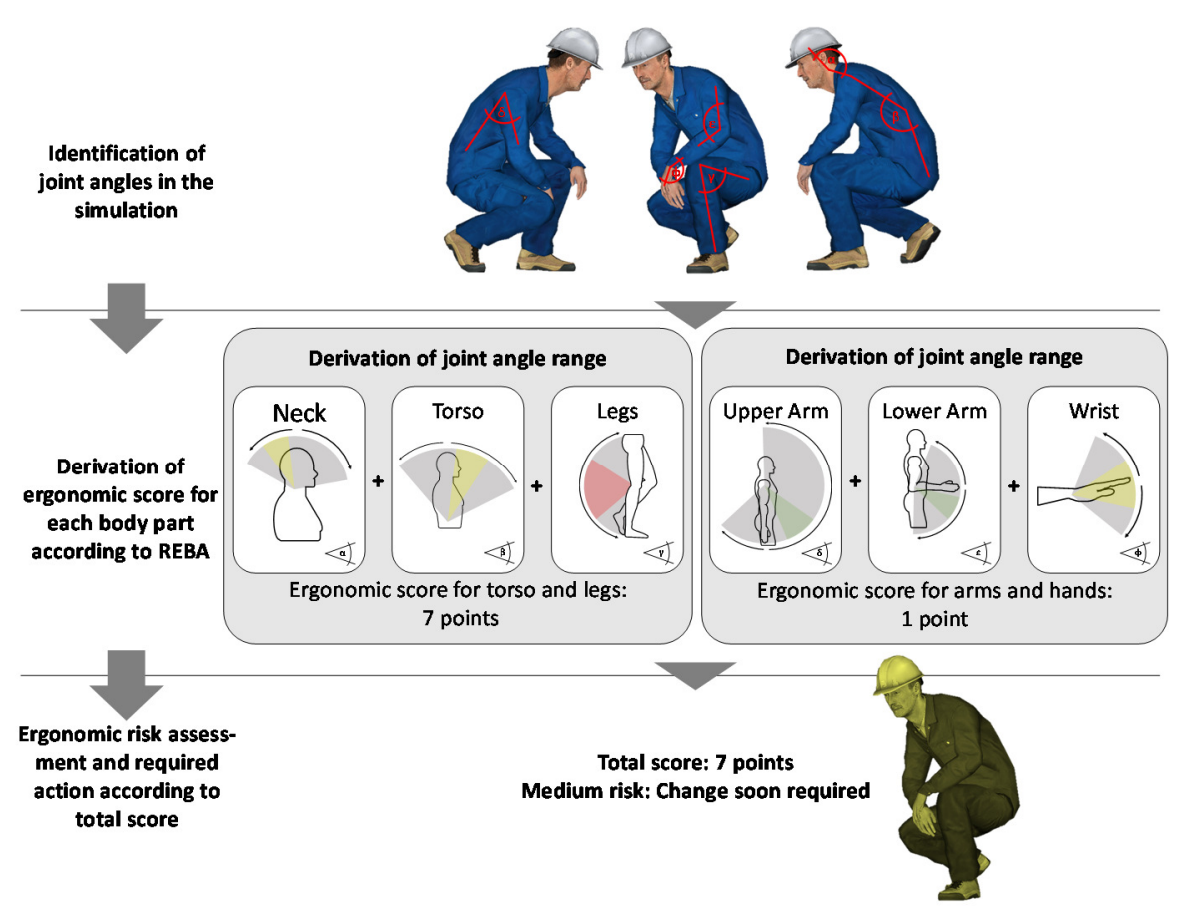

Fig. 2. Ergonomic Assessment with REBA by Automatic Observation of Individual Joint Angles

\section{Software Tool}

For the assessment of the individual anthropometric and kinematic parameters (iakp) and the subsequent scaling of the FAMOS DHM-system a software tool was written for the use with a Microsoft Kinect v2 sensor. The tool is based on the Microsoft Kinect Software Development Kit Version 2.0 (SDK). It handles the Kinect streams, calculates segment lengths, captures range of motions for selected joints and exports the data to a comma separated value (CSV) exchange data file. A simple graphical user interface (GUI) offers case specific modules:

\subsection{Anthropometric Parameter Module}

The anthropometric parameter module was used for the assessment of body segment lengths. It is possible to capture up to four different inertial poses, depending on the needs of the later application. For the current study, the T-Pose was used. For each pose there are three buttons: record, reset and data export. A skeleton is drawn alongside, for an optical consistency check. After successful recording, the table underneath the record buttons shows the results of the body segments for each captured frame, as well as mean values and standard deviations. If data is available, the red rectangle underneath the corresponding pose picture is colored green (figure 3). The data can be exported and is further stored in memory for subsequent data computing. 


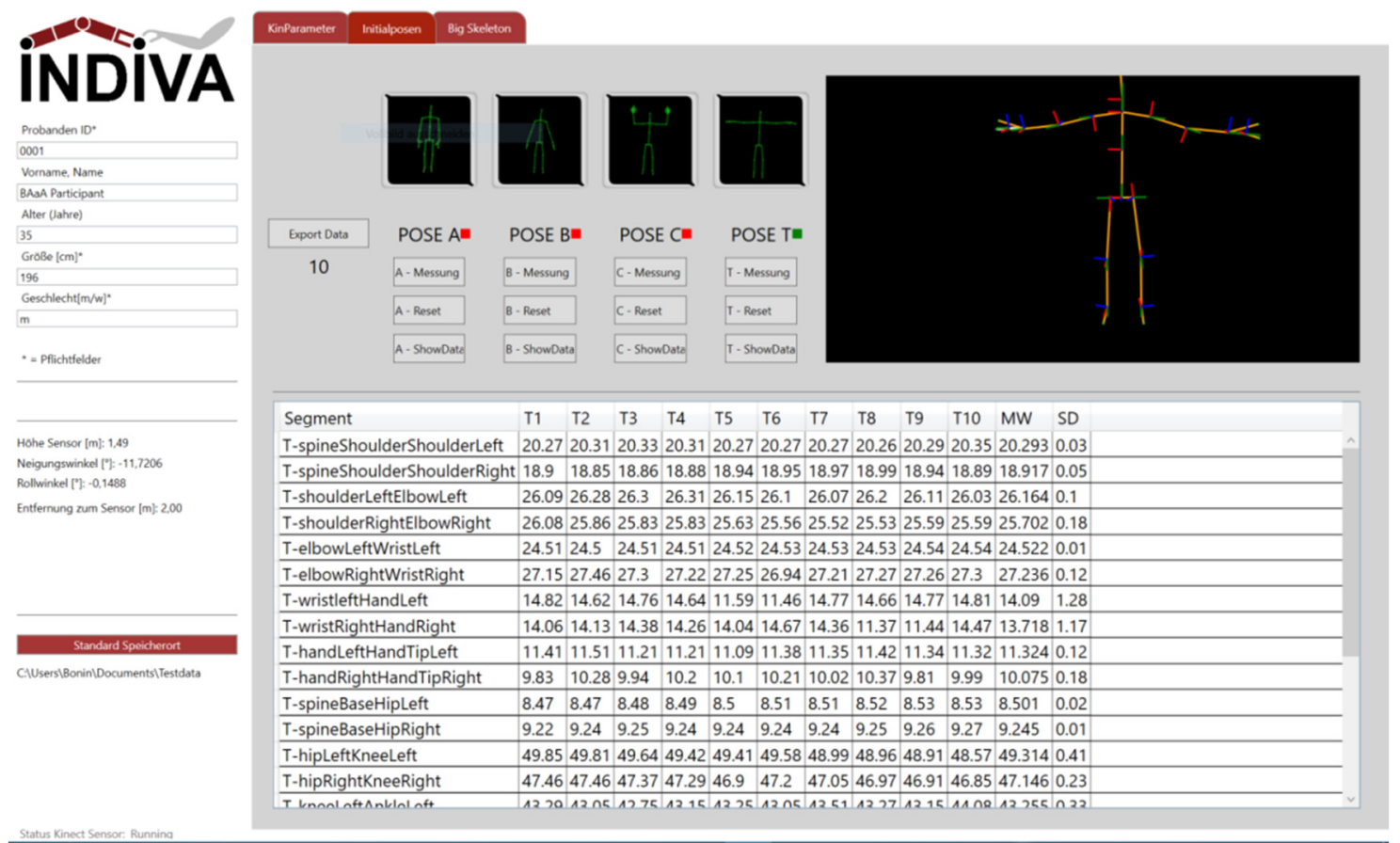

Fig. 3. Screenshot from the Software Tool GUI, Anthropometric Parameter

\subsection{Kinematic Parameter Module}

The kinematic parameter module offers the possibility to record a defined range of motion per joint separately. For each joint and motion, an info text is displayed, how the participant should be placed in front of the camera. For a simple optical check the parts of the skeleton which are necessary for the assessment are displayed on top of the video. A button "Calibrate 0 Angle" is available if a participant is not able to reach the neutral position (figure 4). The recordable motion directions for each joint are listed in Table 1.

Table 1. Assessed Joint ROM Movements

\begin{tabular}{|l|l|}
\hline Joint & Direction \\
\hline Cervical Spine (Head / Neck) & Flexion / Extension \\
& Lateral Flexion \\
& Rotation \\
\hline Trunk & Flexion / Extension \\
& Lateral Flexion \\
& Rotation \\
\hline Shoulder & $\begin{array}{l}\text { Flexion / Extension } \\
\text { Adduction / Abduction / Elevation } \\
\end{array}$ \\
Horizontal Flexion \\
\hline Elbow & Flexion / Extension \\
\hline Wrist & Radial / Ulnarabduction \\
& Palmarflexion / Dorsalflexion \\
\hline Hip & Flexion / Extension \\
& Adduction / Abduction \\
\hline Knee & Flexion / Extension \\
\hline Ankle & no reliable measures \\
\hline
\end{tabular}

The joint angles are calculated with the C\# internal Math.Atan2() function. With this function the angle between two points and a reference plane can be calculated. For example to obtain the left elbow angle, the angle between the lower arm (segment between left elbow joint and left wrist joint) and the $X Z$-plane was calculated as well as the angle between the upper arm (segment between left shoulder and left elbow) and the XZ-plane. Taking the movement direction into account, the values were subtracted from each other to obtain the absolute angle between these two segments within the defined plane. For each motion the position of the participant in front of the sensor was predefined. 
When using the standard skeleton [15], the cervical measures did not show reliable values. With the implementation of the Kinect HD Face application programming interface (API) it was possible to assess additional 3D points of the face as well as a calculated head pivot point. The API offers the face orientation as quaternion [16]. The open source Microsoft.Kinect.Face demo-application delivers a function called "ExtractFaceRotationInDegrees" which was used with the Face HD orientation quaternion in the present scenario. The function returns rotation values for flexion and extension ("pitch"), rotation ("yaw") and lateral flexion ("roll") in degrees.

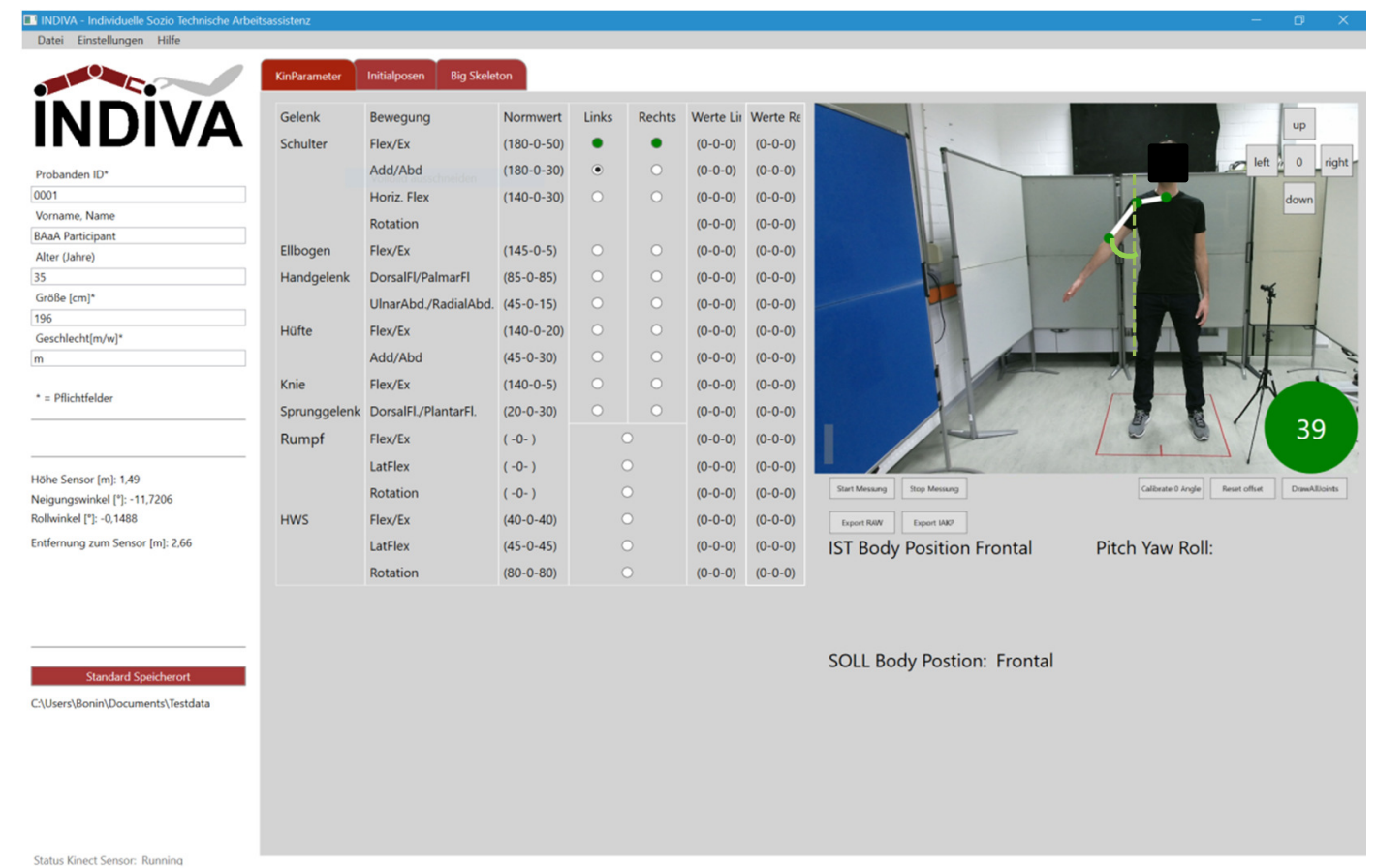

Fig. 4. Modified Screenshot of the Kinematic Parameter Module

\subsection{Local Coordinate View Module, Data Exchange Format}

To facilitate the interpretation of the transformation from local joint orientations between the SDK and the DHM-system, it is possible to display the local joint orientations on an enlarged skeleton model. Each joint can be selected separately from a checkable menu entry. For each possible motion direction a green, red and blue colored line is drawn, the origin located within the assumed joint center. The green line $(Y)$ always matches the skeleton in parent bone direction. The blue line $(Z)$ represents the normal to the bone and the red line $(X)$ the binormal, perpendicular to bone and normal [17].

For the exchange of data between the software tool and the DHM a CSV data format was developed. The file extension *.iakp is an abbreviation for individual anthropometric and kinematic parameters. The format stores information about absolute joint positions, range of motion for each joint in $\mathrm{X}, \mathrm{Y}$ and $Z$ direction as well as the segment length in meter. The file consists of eight columns: Node, T-Pose, algebraic sign, joint axis, min joint value, max joint value, parent node and distance to parent node. The rows represent the measured data, three rows are needed for each joint to represent the translational $\mathrm{X}, \mathrm{Y}$ and $\mathrm{Z}$ range of motions. With a special nodemap the body joints can be assigned between the two systems as well as the segment lengths for subsequent scaling. The absolute joint positions might be used as a control function to reinsure an appropriate scaling of the DHM. The following two columns ensure a good compatibility to other systems, as algebraic sign and rotation order can be modified for each joint separately. The min and max joint values represent the possible motion in the specific direction. If a joint has restricted degrees of freedom the values of the according motion direction are set to zero. The parent node is calculated from the hierarchical Kinect v2 skeleton, the distances between parent and child are displayed in meters.

\section{Method Validation Study}

For the validation of the iakp measurements, the digital assessed values are compared to manual measures. The results are preliminary as the data collection was still ongoing while writing this paper. At this stage, seventeen healthy men, aged between 18 and 34 participated in this study. The manual 
measures were taken from one experienced observer with an anthropometer, goniometer, inclinometer and sliding caliper. For the digital assessment the previously described software tool was used. The validation study consists of two parts, within the first part only the segment length was compared and in the second part the range of motions were addressed. Within this paper, the results of the first part will be presented.

\subsection{Digital Assessment of Segment Length}

The individual anthropometric body measures were captured in a static body position. For the calculation of absolute body measures, the origin from the Kinect v2 was transformed to a point perpendicular underneath the sensor on floor level. The sensor alignment was translated compensating for pitch and roll by using the Vector4 element "FloorClipPlane". The FloorClipPlane is delivered with every bodyframe from the SDK. This Vector4 element consists of four elements; the first three elements $(X, Y, Z)$ representing the normal to the ground plane, the fourth element $(W)$ equals the height of the sensor above the floor level. In the current study a T-Pose was chosen as reference for data exchange like it is commonly used in gaming industries and avataring: Participants facing the sensor, standing straight, legs are close together, arms are elevated straight $90^{\circ}$ to the side, palms facing downwards.

To calculate the segment length, the software defines a segment as a three dimensional vector (Vector3D) between the 3D coordinates of the parent joint and corresponding child joint within the hierarchical skeleton structure (figure 5).

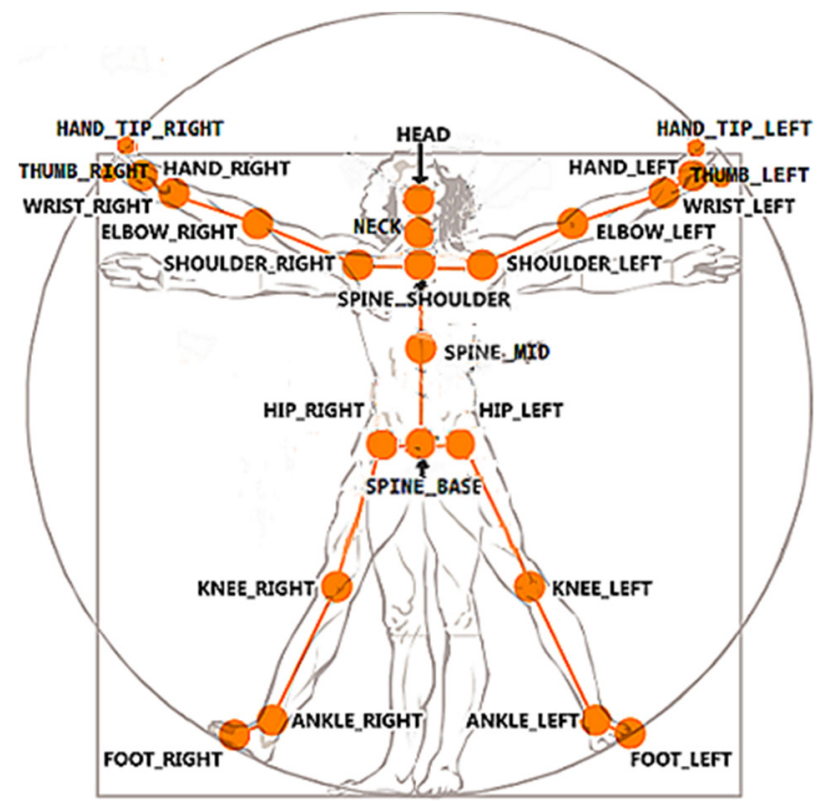

Fig. 5. Standard Microsoft Kinect v2 Skeleton [15]

For each defined Vector3D segment the System.Media.Media3D Vector3D.Length method was used to calculate the distance between parent and child. The inertial pose was recorded at two frames per second, with auto stop after ten captured frames. The values are then exported to a CSV file.

\subsection{Manual Assessment of Segment Length}

For the manual assessment of segment length only the right body side was measured. For the comparison to the digital assessed values it was necessary to take into account that the digital values are derived from an "inner-skeleton" which calculates the segment lengths from one assumed joint center to the other. This means that some of the distances couldn't be assessed directly from the surface and had to be recalculated to compare the manual values with the digital assessed values from the 3D skeleton. For example the horizontal distance between the two hip joint centers was calculated with a greater-trochanter based location method as described in [18]. The shoulder joint centers were calculated acromion based as described in [19]. Table 2 describes the evaluated segments and corresponding additional literature. 
Table 2: Measurement Details for the Manual Assessment of Segment Lengths

\begin{tabular}{|l|l|l|}
\hline Segment length & Measurement details & Literature \\
\hline $\begin{array}{l}\text { Upper arm } \\
\text { length: }\end{array}$ & $\begin{array}{l}\text { (acromion to head of radius - 10,4\%) - Distance between head of } \\
\text { radius and lateral epicondyle }\end{array}$ & {$[1,19,20]$} \\
\hline Forearm length: & $\begin{array}{l}\text { Distance between farthest proximal point of head of radius and ulnar } \\
\text { styloid }\end{array}$ & {$[20]$} \\
\hline Hand length: & Radial styloid to the farthest distal point of dactylion III & {$[1]$} \\
\hline Thigh length: & Trochanter to lateral condyle of femur & \\
\hline Shank length: & Upper part of the head of the fibula to lateral malleolus & {$[1,20]$} \\
\hline Foot length: & From acropodium to farthest distal point of lateral malleolus & {$[20]$} \\
\hline Shoulder width: & From ipsilateral acromion to contralateral acromion & {$[18]$} \\
\hline Hip width: & 0,5 * distance of ipsilateral and contralateral greater trochanter & {$[1]$} \\
\hline Neck to Head: & C7 to Frankfurt plane (eye-ear plane horizontal) \\
\hline
\end{tabular}

\section{Results}

As mentioned before, only the first part of the validation study which handles the assessment of anthropometric parameters is presented within this paper. The second part is still ongoing and will be presented separately. To compare the manual and digital assessment method, the absolute differences for each measurement, the mean of differences and standard deviation of mean differences (SDd) were calculated. The differences were calculated as manual values minus digital values. Table 3 shows the mean values of difference and standard deviation of difference for the extremities and Table 4 the mean values of difference and standard deviation of difference for the torso, head and neck. If the values are negative, the digital measurement showed higher values compared to the manual measures. ${ }^{*}=$ recalculated with offset, see chapter $5.2+7,{ }^{* *}=$ not reliable due to scattering foot joint, see chapter 7

Table 3: Mean difference and Standard Deviation of Segment Length, Digital vs. Manual, Part 1: extremities UpperArm = Shoulder_right to Elbow_right, ForeArm = Elbow_right to Wrist_right, Hand = Wrist_right to HandTip_right, Thigh $=$ Hip_right to Knee_right, Foot $=$ Knee_right to Ankle_right.

\begin{tabular}{|l|l|l|l|l|l|l|}
\hline & UpperArm & ForeArm & Hand & Thigh & Shank & Foot $^{\star *}$ \\
\hline mean diff $[\mathrm{cm}]$ & 4.41 & 1.08 & 0.58 & 0.78 & -0.17 & 6.5 \\
\hline SDd & 2.14 & 1.29 & 1.69 & 1.29 & 2.83 & 3.64 \\
\hline
\end{tabular}

Table 4: Mean Difference and Standard Deviation of Segment Length, Digital vs. Manual, Part 2: torso + head+ neck Head $=$ Head to Neck, Neck $=$ Neck to SpineShoulder, Shoulder width $=0.5$ * distance between Shoulder_left and Shoulder_right, Spine $=$ SpineBase to SpineShoulder, Hip = SpineBase to Hip_right.

\begin{tabular}{|l|l|l|l|l|l|}
\hline & Head & Neck & Shoulder width & Spine* & Hip $^{*}$ \\
\hline mean diff $[\mathrm{cm}]$ & -0.41 & -0.41 & -0.69 & 4.78 & -0.35 \\
\hline SDd & 1.71 & 2.27 & 1.46 & 2.98 & 0.53 \\
\hline
\end{tabular}

To visualize the comparison, a Bland-Altmann plot [21] was used (figure 6). The confidence interval was defined by $95 \%$, so that the upper and lower limits were calculated as: mean of differences +1.96 * SDd and mean of difference - 1.96 * SDd. For data points above the mean line, the manual measured segment length values were higher, for data points underneath the mean line, the digital assessed segment lengths values were higher. 


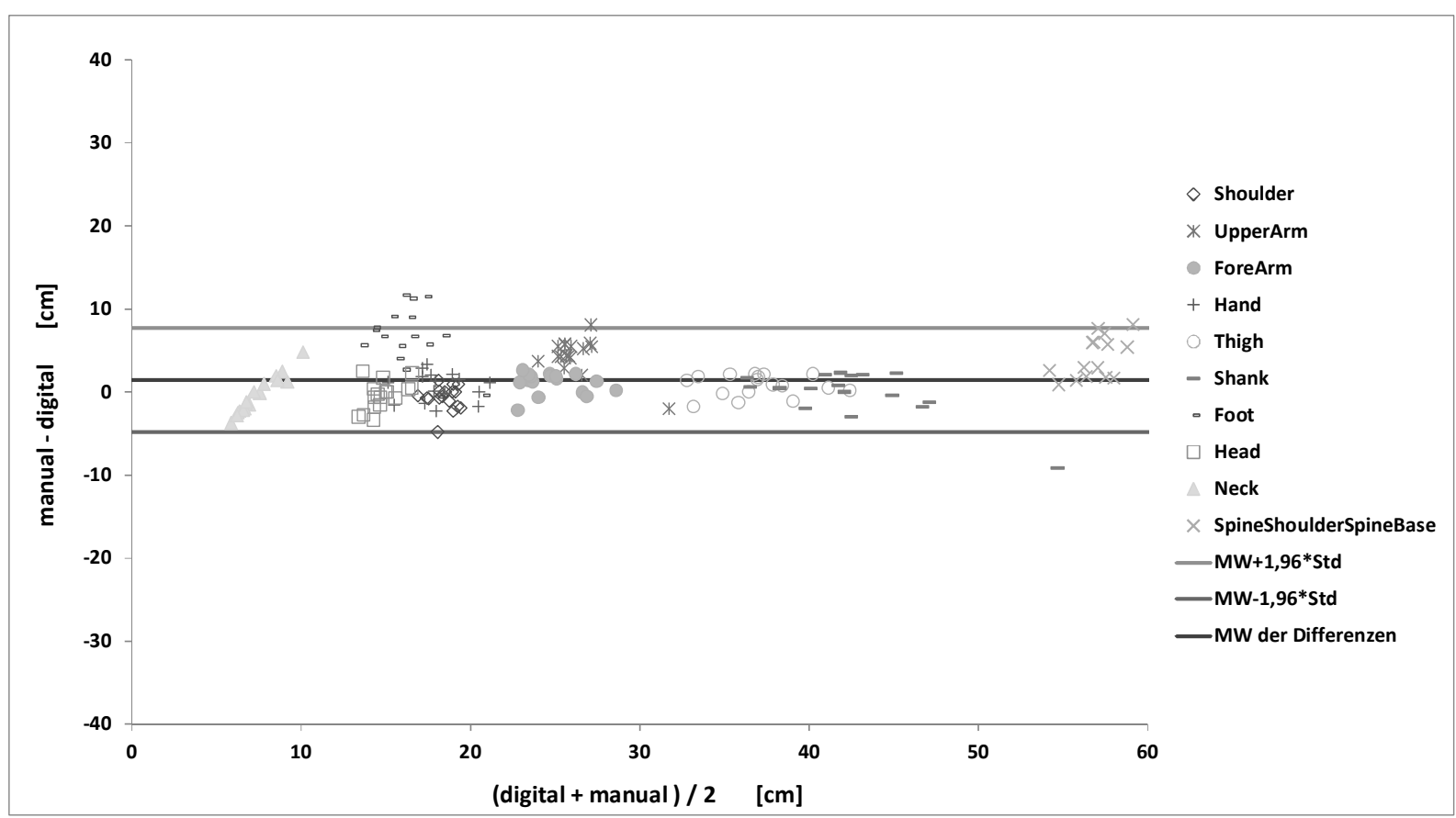

Fig 6. Bland-Altmann Plot Digital vs. Manual Assessment

\section{Discussion}

The values presented before show ambiguous results, depending on the specific body area. For the shoulder joint center the used method and related offset taken from [19] seem to be inaccurate for our setup. The recalculated manual measures of the upper arm are higher compared to the digital values. This means that the assumed shoulder joint center of the Kinect skeleton doesn't match the recalculated joint point from the shoulder. However, the values show a relative constant and reproducible offset so that further data analysis with a greater pool of participants will offer the possibility to calculate new reliable offsets. Further, the absolute height of the SpineShoulder joint point was referenced with the same offset from acromion height as the shoulder joint, referring to the Microsoft Kinect v2 skeleton where the joints ShoulderLeft, SpineShoulder and ShoulderRight are on one line (figure 5). Because of this fact, the mean differences of the spine segment lengths were influenced as well. Another body part with high deviation was the foot length. The values are very inaccurate with high standard deviations. We assume that this is most likely a sensor related issue because it was optical observable during the validation study that the skeleton joint point was randomly scattering even in static positions. The remaining values showed a good agreement between digital and manual assessment with mean differences from $-0.17 \mathrm{~cm}$ (Shank, SDd 2.8) to a maximum of $1.08 \mathrm{~cm}$ (ForeArm, SDd 1.29). One big advantage of the digital tool over the manual measurement is the assessment time. For the assessment with the tool, a total measurement time underneath two minutes was achievable, while the manual assessment took about fifteen to twenty minutes in total. In conclusion, the digital assessment with the optical markerless motion capturing system offered constant and reproducible values (except from the foot values). The values are applicable for a subsequent scaling of the DHM-system so that individual peculiarities will have a remarkable effect on the adjusted DHM.

The scaling worked well but still the skeleton of the FAMOS DHM was only scaled based on internal segment lengths. No skin or circumference measures were assessed. In the current process a standard mesh was applied around the scaled skeleton. Further investigations should include more parameters like the body shape. These values might be extracted from the raw 3D point cloud of the depth data stream.

In chapters two and three the industrial demand for HRC-workplaces was shown as well as a workflow for a holistic and humane design of these collaborative scenarios. The results from the anthropometric assessment validation were presented. The data format was defined so that each possible motion direction of the skeleton gets min and max joint rotation limits. The current storage of the kinematic parameters is in line with the neutral-0 method. Right now the iakp file is exported with standard kinematic values. The current research focusses on the implementation of the assessed kinematic parameters within the FAMOS DHM-system. A translation between the local coordinate system of the Kinect skeleton and the FAMOS skeleton with a recalculation of the neutral-0 values to possible joint 
rotations in FAMOS are in work so the results will be validated and published in the near future. The first preview with only a small sample size seems to promise good results.

With the current level of development, the assessment, the ergonomic simulation and the adjustment of the robot trajectories is working. But still, the simulation and adjustment of the robot trajectories is performed offline. For extended use in real applications the workflow, computing and adjustment time needs to be improved, to work towards automated real-time adjustments.

\section{Conclusion / Outlook}

Within this paper a method and the corresponding validation study is described, to asses individual anthropometric and kinematic parameters with a markerless motion capturing system. Further, use cases for the subsequent computing of the assessed data as well as a workflow for the enhancement of human centered prospective workplace design was presented. However the technology is still at an early stage of development. Right now, the computing of the ergonomic simulation and calculation of appropriate robot trajectories is still time consuming and is therefore performed as offline calculation. Further research should focus on the enhancement of the robot trajectory programming to work towards real time adjustments. As the topic of direct human-robot-collaboration is at a comparatively early stage of development, the presented method is a first step towards a further individualization and improvement of safe, humane and competitive workplaces. But even if the tool produced reliable data it cannot be used automated and is not able to replace human judgement, knowledge or sense of responsibility.

\section{Acknowledgement}

The presented results are part of the research project INDIVA. The research \& development project is funded by the German Federal Ministry of Education and Research (BMBF) and attended by the VDI/VDE-IT GmbH.

A special thanks goes to Mr. Felix Lunemann who did a great job with the measurements for the validation study.

\section{References}

[1] International Organization for Standardization, "ISO 7250," in Basic human body measurements for technological design, ed.

[2] C. Ryf and A. Weymann, "The neutral zero method-a principle of measuring joint function," Injury, vol. 26, pp. 1-11, 1995.

[3] C. Thomas, L. Stankiewicz, A. Grötsch, S. Wischniewski, J. Deuse, and B. Kuhlenkötter, "Intuitive Work Assistance by Reciprocal Human-robot Interaction in the Subject Area of Direct Human-robot Collaboration," Procedia CIRP, vol. 44, pp. 275-280, 2016.

[4] C. Brandl, D. Bonin, A. Mertens, S. Wischniewski, and C. M. Schlick, "Approaches for the digitization of ergonomic analyses and intervention using the example of markerless motion capture of working postures during work tasks in production," Zeitschrift für Arbeitswissenschaft, vol. 70, pp. 89-98, 2016.

[5] D. Bonin and S. Wischniewski, "Evaluating the accuracy of markerless body tracking for digital ergonomic assessment using consumer electronics," in 19th Triennial Congress of the Inernational Ergonomics Association, Melbourne, Australia, 2015.

[6] S. Wischniewski, U. Hold, and M. Lehmann, "Ergonomische Analyse menschlicher Bewegungsabläufe mittels Spielekonsolen-Technologie - Aktuelle Möglichkeiten und Grenzen.," in Bericht zum 58. Kongress der Gesellschaft für Arbeitswissenschaften, Universität Kassel, 2012, pp. 123-126.

[7] B. Bonnechère, B. Jansen, P. Salvia, H. Bouzahouene, L. Omelina, F. Moiseev, et al., "Validity and reliability of the Kinect within functional assessment activities: Comparison with standard stereophotogrammetry," Gait \& Posture, vol. 39, pp. 593-598, 1// 2014.

[8] J. A. Diego-Mas and J. Alcaide-Marzal, "Using Kinect sensor in observational methods for assessing postures at work," Appl Ergon, vol. 45, pp. 976-85, Jul 2014.

[9] T. Dutta, "Evaluation of the Kinect ${ }^{\mathrm{TM}}$ sensor for 3-D kinematic measurement in the workplace," Applied Ergonomics, vol. 43, pp. 645-649, 7// 2012.

[10] T. Nguyen, M. Kleinsorge, and J. Kruger, "ErgoAssist: An assistance system to maintain ergonomic guidelines at workplaces," in Emerging Technology and Factory Automation (ETFA), 2014 IEEE, 2014, pp. 1-4. 
[11] F. Busch, "Ein Konzept zur Abbildung des Menschen in der Offline-Programmierung und Simulation von Mensch-Roboter-Kollaborationen.," Shaker, Aachen, Germany, 2015.

[12] L. Stankiewicz, C. Thomas, J. Deuse, and B. Kuhlenkötter, "Application of Customizable Robot Assistance Systems to Compensate Age-Related Restrictions of the Musculoskeletal System for Assembly Workplaces," Applied Mechanics \& Materials, vol. 840, 2016.

[13] F. Busch, J. Hartung, C. Thomas, S. Wischniewski, B. Dortmund, J. Deuse, et al., "Individualisierte Arbeitsassistenz in der Produktion," Industrie Management - Demografische Veränderung der Arbeitswelt, p. 7, 03/2013.

[14] F. Liebers, C. Brendler, and U. Latza, "Berufsspezifisches Risiko für das Auftreten von Arbeitsunfähigkeit durch Muskel-Skelett-Erkrankungen und Krankheiten des Herz-Kreislauf-Systems. Bestimmung von Berufen mit hoher Relevanz für die Prävention," ed. Dortmund, Berlin, Dresden: Bundesanstalt für Arbeitsschutz und Arbeitsmedizin (BAuA), 2016.

[15] Microsoft Developer Network. (2016). Kinect v2 Joint Type Enumeration, Access Date 23.09.2016. Available: https://msdn.microsoft.com/en-us/library/microsoft.kinect.jointtype.aspx

[16] Microsoft Developer Network. (2016). Access Date 21.09.2016. Available: https://msdn.microsoft.com/de-de/library/dn785525.aspx

[17] C. Sirignano. (2014). Kinect v2.0 Preview SDK JointOrientation vs BoneOrientation. Available: https://social.msdn.microsoft.com/Forums/en-US/31c9aff6-7dab-433d-9af9-59942dfd3d69/kinect -v20-preview-sdk-jointorientation-vs-boneorientation?forum=kinectv2sdk

[18] J. T. Weinhandl and K. M. O'Connor, "Assessment of a greater trochanter-based method of locating the hip joint center," J Biomech, vol. 43, pp. 2633-6, Sep 172010.

[19] P. de Leva, "Joint center longitudinal positions computed from a selected subset of Chandler's data," Journal of Biomechanics, vol. 29, pp. 1231-1233, 1996/09/01 1996.

[20] B. Flügel, H. Greil, and K. Sommer, Anthropologischer Atlas : Grundlagen und Daten, Deutsche Demokratische Republik, 1. Aufl. ed. Berlin: Verl. Tribüne, 1986.

[21] J. Martin Bland and D. Altman, "STATISTICAL METHODS FOR ASSESSING AGREEMENT BETWEEN TWO METHODS OF CLINICAL MEASUREMENT," The Lancet, vol. 327, pp. 307-310, 1986/02/08 1986. 